S. Takenaka

Nagoya Math. J.

Vol. 123 (1991), 1-12

\title{
INTEGRAL-GEOMETRIC CONSTRUCTION OF SELF-SIMILAR STABLE PROCESSES
}

\author{
SHIGEO TAKENAKA \\ Dedicated to Professor H. Nomoto for his 60th birthday
}

\section{§1. Introduction}

Recently, fractional Brownian motions are widely used to describe complex phenomena in several fields of natural science. In the terminology of probability theory the fractional Brownian motion is a Gaussian process $\{X(t): t \in \mathbf{R}\}$ with stationary increments which has a self-similar property, that is, there exists a constant $H$ (for the Brownian motion $H=1 / 2$, in general $0<H<1$ for Gaussian processes) called the exponent of self-similarity of the process, such that, for any $c>0$, two processes $\{X(c t): t \in \mathbf{R}\}$ and $\left\{c^{H} X(t): t \in \mathbf{R}\right\}$ are subject to the same law (see [10]).

Let us consider the following generalization of fractional Brownian motions.

Definition. A real-valued stochastic process $\left\{X(\mathfrak{t}) ; \mathfrak{t} \in \mathbf{R}^{d}\right\}$ is called an $(\alpha, H)$-process if the following three conditions are fulfilled.

i) $\{X(\mathrm{t})\}$ is a symmetric stable family of index $\alpha$. That is, any finite linear combination $\sum a_{i} X\left(\mathbb{t}_{i}\right)$ is subject to a symmetric stable law of index $\alpha$, equivalently, the characteristic function $\mathbf{E}\left[\exp \left\{i z \sum a_{i} X\left(\mathrm{t}_{i}\right)\right\}\right]$ is written as $\exp \left(-\kappa|z|^{\alpha}\right)$ with a positive constant $\kappa=\kappa\left(a_{i}, \mathfrak{t}_{i} ; i=1, \cdots, n\right)$.

ii) $\{X(\mathbb{t})\}$ has stationary increments with respect to the action of Euclidean solid motion. That is, $X(g \mathfrak{t})-X(g \mathbb{O})$ is subject to the the same law with $X(\mathfrak{t})$ for any $\mathfrak{t} \in \mathbf{R}^{d}$ and $g \in M(d)$, where $M(d)$ denotes the group of $d$-dimensional Euclidean solid motions. Note that $X(\mathbb{D})$ is automatically equal to 0 , a.e. (Take $g=i d$, the identity, and $t=D$ ).

iii) $\{X(\mathbb{t})\}$ is a self-similar process of exponent $H$. That is, for any $c>0$, the process $\left\{X^{c}(\mathfrak{t}) \equiv X(c \mathfrak{t}) ; \mathfrak{t} \in \mathbf{R}^{d}\right\}$ has the same finite-dimensional distributions as $\left\{c^{H} X(\mathbb{t})\right\}$.

Received February 28, 1990. 
The $\mathbf{R}^{1}$-parameter symmetric (starting from 0 ) stable process of index $\alpha, 0<\alpha \leq 2$, with stationary independent increments is an $(\alpha, 1 / \alpha)$-process. Fractional Brownian motions are examples of $(\alpha, H)$-processes of $\alpha=2$, $0<H<1$ and $d=1$. For the Gaussian case, $\alpha=2$, the author has already obtained the $(2, H)$-process for $0<H<1$ for any dimension $d$ (see [12]). This paper is a direct generalization of the former results on Gaussian processes to stable processes. An integral geometric method is used in the construction of these processes. We will see the differences and the similarity between the Gaussian case and the stable case which share the same geometrical structure. Especially, the determinism described in the last section suggests us that there is quite good possibility to extend deep results on the Gaussian system to the stable processes constructed by the integral geometry, and that this type of processes will give us good examples on the theory of stable processes. In fact, the author and K. Kojo have already obtained some results on the canonical representations of a stable analogues of Lévy's $M_{t}$-processes ([6]).

After showing a necessary condition on $(\alpha, H)$ under which $(\alpha, H)$ process exists $(\S 2)$, we will make a series of examples of $(\alpha, H)$-processes using integral geometry ( $\S 3)$. In $\S 4$ we will calculate 2-dimensional distributions and we will see that our examples make a new class of $(\alpha, H)$ processes. The set of processes which are constructed by a common integral-geometric structure is called a conjugate class. In the last section, some properties of conjugate classes are discussed.

In the construction of examples we use an $\alpha$-stable random measure corresponding to a measure space $(E, \mathscr{B}, \mu)$.

Definition. A family of random variables $\mathscr{Y}=\{Y(B) ; B \in \mathscr{B}, \mu(B)<$ $\infty\}$ is called an $\alpha$-stable random measure corresponding to $(E, \mu)$ if

i) $\mathbf{E}[\exp (i z Y(B))]=\exp \left(-\mu(B)|z|^{\alpha}\right)$.

ii) $Y\left(B_{j}\right), j=1, \cdots, n$, are mutually independent if $B_{j} \cap B_{k}=\varnothing, j \neq k$.

iii) $Y\left(\cup B_{j}\right)=\sum Y\left(B_{j}\right)$, a.e., for any disjoint family $\left\{B_{j} ; j=1,2, \cdots\right\}$.

It is easy to see that, for any $\sigma$-finite measure space, the corresponding $\alpha$-stable random measure exists (use the method of construction by inductive limit. cf. [1]).

\section{§ 2. Existence conditions and known results}

2-1. Suppose that $\left\{X(\mathfrak{t}), \mathfrak{t} \in \mathbf{R}^{d}\right\}$, is an $(\alpha, H)$-process. We first note 
that from the definition $X(\mathbb{D})$ should be equal to 0 , a.e. (see ii) of the definition). The case of 1-dimensional parameter, $d=1$, the following are known. Let $\{X(t) ; t \in \mathbf{R}\}$ be an $H$-self-similar process with stationary increments. If $H<0$, then $X(t)=0$ a.s. And if $H=0, X(t)=X(0)$ a.s. In addition, if $\{X(t) ; t \in \mathbf{R}\}$ is $\alpha$-stable, then $(\alpha \wedge 1) H \leq 1$ ([5]). On the other hand, W. Verraat proves that there is no 1-self-similar process with stationary increments having the finite first moment except the degenerate process $\{X(t)=t X(1) ; t \in \mathbf{R}\}$ ([14]).

Let us consider an $\mathbf{R}^{a}$-parameter $(\alpha, H)$-process and its restriction of parameters to a 1-dimensional linear subspace. It is easy to see that this restriction is an $\mathbf{R}^{1}$-parameter $(\alpha, H)$-process. Thus, the above facts can be observed also for our $(\alpha, H)$-process without any changes in their proofs. We thus have

Proposition 1. A necessary condition of the existence of non-trivial $(\alpha, H)$-process is

$$
(\alpha, H) \in\{H<1,0<\alpha \leq 2\} \cup\left\{H \leq \frac{1}{\alpha}, 0<\alpha \leq 2\right\} .
$$

We will see that there exist some examples of $(\alpha, H)$-processes for every $(\alpha, H)$ in the above area. Thus the condition in Proposition 1 is also sufficient.

2-2. For the first area $\{H<1\} \cap\{0<\alpha \leq 2\}$ and $d=1$, the following examples are discussed in several papers ([2], [4], [5], [8], [10], [13]).

Theorem 2. For a fixed $\alpha, 0<\alpha \leq 2$, set

$$
F(\mathfrak{t}, \mathrm{x}) \equiv|\mathrm{x}|^{H-(d / \alpha)}-|\mathrm{x}-\mathfrak{t}|^{H-(d / \alpha)}, \quad \mathfrak{t}, \mathrm{x} \in \mathbf{R}^{d},
$$

where $|\cdot|$ denotes the Euclidean norm. Then if $0<H<1$ and $H \neq d / \alpha$,

(i ) $F(\mathfrak{t}, \cdot) \in L^{\alpha}\left(\mathbf{R}^{d}, d x\right)$, and

(ii) the process

$$
X(\mathfrak{t} ; \omega) \equiv \int_{\mathbf{R}^{d}} F(\mathfrak{t}, \mathrm{x}) d Z_{\alpha}(\mathrm{x} ; \omega)
$$

is an $(\alpha, H)$-process, where $\left\{Z_{\alpha}(\cdot)\right\}$ is a symmetric $\alpha$-stable random measure corresponding to the Lebesgue measure on $\mathbf{R}^{d}$. 


\section{§ 3. Construction using integral geometry}

The $(2,1 / 2)$-process is nothing but the multi-parameter Brownian motion which is defined by P. Lévy ([7]). It is known that this process can be constructed using integral geometry as below ([3], [7]).

Consider the set $\mathbb{E}$ of the totality of hyperplanes of co-dimension 1 in $\mathbf{R}^{d}$. The group $M(d)$ of Euclidean solid motions acts on $\mathbb{E}$ in the natural manner. Let $\mu$ be the invariant measure on $\mathbb{E}$ with respect to this action of $M(d)$. For any $\mathbf{t} \in \mathbf{R}^{d}$, set

$$
S_{\mathrm{t}} \equiv\{L \in \mathbb{E} ; L \text { separates the point } \mathrm{t} \text { and the origin } \mathbb{D}\} \text {. }
$$

Denote a Gaussian random measure corresponding to $(\mathbb{E}, \mu)$ as $\mathscr{Y}=\{Y(\cdot)\}$, We have

THEOREM $3([3])$.

(i) $\mu\left(S_{\mathrm{t}}\right)<\infty$, for any $\mathrm{t}$.

(ii) The process defined by

$$
X(\mathrm{t}) \equiv Y\left(S_{\mathfrak{t}}\right)
$$

is an $\mathbf{R}^{d}$-parameter Brownian motion.

The triplet $\left\{(\mathbb{E}, \mu), G, S_{\mathbf{t}}\right\}$ is essential to the above construction. Here a group $G-M(d)$ for the above case-acts on a family $\mathbb{E}$ of subsets of $\mathbf{R}^{d}, \mu$ is the invariant measure on $\mathbb{E}$ and $S_{t}$ is a measurable subset of $\mathbb{E}$ whose elements separate the origin $\mathbb{D}$ and the point $\mathrm{t}$.

In this paper we are interested in $(\alpha, H)$-process. We need to extend the group $G$ and the set $\mathbb{E}$. At first, let us fix $\mathbb{E}$ as hyperplanes and let us consider an extension of $G$. The group $G$ may be taken as the Euclidean similarity group $M(d) \times \mathbf{R}_{+}$, the direct product of $M(d)$ and the group $\mathbf{R}_{+}$ of homothetic transforms. This group acts on the set of all hyperplanes of co-dimension 1 in the natural manner. The action of the homothetic part satisfies the relation

$$
S_{c t}=c S_{t}, \quad c \in \mathbf{R}_{+} .
$$

And the measure $\mu$ satisfies

$$
\mu\left(c S_{t}\right)=c \mu\left(S_{t}\right)=c^{\beta} \mu\left(S_{t}\right), \quad \beta=1 .
$$

By the same discussion we can easily obtain the same relation around the covariance of the process (see the proof of Theorem 4), we can easily 
see that the Brownian motion is a $(2,1 / 2)$-process. If we employ an $\alpha$-stable random measure, $0<\alpha<2$, instead of Gaussian random measure, we obtain an $(\alpha, 1 / \alpha)$-process. In the case $d=1$, this process is well known as an $\alpha$-stable process with independent stationary increments. Thus, if we want to consider general $(\alpha, H)$-processes, we have to generalize also the set $\mathbb{E}$. Note that, the measure $\mu$ is not invariant but homothetic under the homothetic action of $\mathbf{R}_{+}$.

A hyperplane may be considered as a circle of radius $\infty$. Now it is natural to take \{all spheres of co-dimension 1 \} as a candidate of $\mathbb{E}$ in our construction of $(\alpha, H)$-processes. Let us introduce a coordinate system in $\mathbb{E}$;

$E \equiv\left\{\left(x_{0}, \mathrm{x}\right) ; x_{0} \in \mathbf{R}_{+}, \mathrm{x} \in \mathbf{R}^{d}\right\}$, where the element $\left(x_{0}, \mathrm{x}\right)$ is considered as the sphere of center $\mathrm{x}$ and radius $x_{0}$.

The action $(g, c) \in M(d) \times \mathbf{R}_{+}$on $\mathbb{E}$ is defined as

$$
(g, c)\left(x_{0}, \mathrm{x}\right) \equiv\left(c \cdot x_{0}, c \cdot g \mathrm{x}\right) \text {. }
$$

The stationarity (ii) suggests us that the measure $\mu$ on $\mathbb{E}$ may be taken invariant under the action $M(d)$. Taking the self-similarity into account, let us take $\mu$ as

$$
d \mu_{\beta}\left(x_{0}, \mathrm{x}\right)=x_{0}^{\beta-d-1} d x_{0} d \mathbf{x} .
$$

The set $S_{\mathrm{t}}$ is defined as

$$
\begin{aligned}
S_{\mathbf{t}} & \equiv\{(d-1) \text {-spheres which separate the origin and the point } \mathfrak{t}\} \\
& \equiv\left\{\left(x_{0}, \mathbf{x}\right) ;\|\mathbf{x}\| \leq x_{0}\right\} \triangle\left\{\left(x_{0}, \mathbf{x}\right) ;\|\mathbf{x}-\mathbf{t}\| \leq x_{0}\right\}
\end{aligned}
$$

where $\Delta$ means symmetric difference.

For the convenience of the following discussion, let us introduce a notation

$$
\tilde{S}_{\mathrm{t}}=\{(x, \mathrm{x}) ;\|\mathrm{x}-\mathfrak{t}\| \leq x\}, \quad \text { then } S_{\mathrm{t}}=\tilde{S}_{\mathrm{t}} \triangle \tilde{S}_{0}
$$

We have

Theorem 4 (for the Gaussian case see [12]).

1) $\mu_{\beta}\left(S_{\mathfrak{t}}\right)<\infty$ if and only if $0<\beta<1$.

2) The process $\left\{X_{\alpha, \beta}(\mathfrak{t}) \equiv Y_{\alpha}\left(S_{\mathfrak{t}}\right) ; \mathfrak{t} \in \mathbf{R}^{d}\right\}$, for $0<\beta<1$, is an $(\alpha, \beta / \alpha)$ process, where $\mathscr{Y}_{\alpha}=\left\{Y_{a}(\cdot)\right\}$ is an $\alpha$-stable random measure corresponding to $\left(\mathbb{E}, \mu_{\beta}\right)$.

Proof. "If" part of the proof 1) is easily seen, so we omit it. To 
prove "only if" part, let us suppose $\mu_{\beta}\left(S_{t}\right)<\infty$ for a $\beta>1$. By the proof of 2) below there exists an $(\alpha, \beta / \alpha)$-process for the parameter $H>1 / \alpha$. This contradicts the result of Proposition 1. Thus we have proved the "only if" part if $\mu_{\beta}\left(S_{\mathfrak{t}}\right)<\infty$ and $\beta<1$.

2) Because $\mu_{\beta}\left(S_{\mathfrak{t}}\right)$ is finite, the process $\left\{X_{\alpha, \beta}(\mathbb{t})\right\}$ is well defined. Recall that $S_{\mathrm{t}}=\tilde{S}_{\mathrm{t}} \triangle \tilde{S}_{0}$. It holds

$$
\begin{aligned}
X_{\alpha, \beta}(\mathrm{t})-X_{\alpha, \beta}(\mathrm{S})= & Y_{\alpha}\left(\tilde{S}_{\mathrm{t}} \triangle \tilde{S}_{0}\right)-Y_{\alpha}\left(\tilde{S}_{s} \triangle \tilde{S}_{0}\right) \\
= & Y_{\alpha}\left(\left(\tilde{S}_{\mathrm{t}} \cap{ }^{c} \tilde{S}_{0}\right) \cup\left({ }^{c} \tilde{S}_{\mathrm{t}} \cap \tilde{S}_{0}\right)\right)-Y_{\alpha}\left(\left(\tilde{S}_{s} \cap{ }^{c} \tilde{S}_{0} \cup\left({ }^{c} \tilde{S}_{s} \cap \tilde{S}_{0}\right)\right)\right. \\
= & \left\{Y_{\alpha}\left(\left(\tilde{S}_{\mathrm{t}} \cap{ }^{c} \tilde{S}_{\mathbf{s}}\right) \cap{ }^{c} \tilde{S}_{0}\right)-Y_{\alpha}\left(\left(\tilde{S}_{\mathrm{t}} \cap{ }^{c} \tilde{S}_{\mathrm{s}}\right) \cap \tilde{S}_{0}\right)\right\} \\
& +\left\{Y_{\alpha}\left(\left({ }^{c} \tilde{S}_{\mathrm{t}} \cap \tilde{S}_{s}\right) \cap \tilde{S}_{0}\right)-Y_{\alpha}\left(\left({ }^{c} \tilde{S}_{\mathrm{t}} \cap \tilde{S}_{s}\right) \cap{ }^{c} \tilde{S}_{0}\right)\right\}
\end{aligned}
$$

where ${ }^{C} A$ means the complement of the set $A$. As we are concerning the symmetric stable law, the above random variable is subject to the same law with $Y_{\alpha}\left(\tilde{S}_{\mathrm{t}} \cap{ }^{c} \tilde{S}_{\mathrm{s}}\right)-Y_{\alpha}\left({ }^{(} \tilde{S}_{\mathrm{t}} \cap \tilde{S}_{\mathrm{s}}\right)$. And the measure $\mu_{\beta}$ is invariant under the action of $M(d)$. Thus $X_{\alpha, \beta}(\mathrm{t})-X_{\alpha, \beta}(\mathrm{s})$ and $X_{\alpha, \beta}(\mathrm{t}-\mathrm{s})$ are subject to a common stable law. That is, $\left\{X_{\alpha, \beta}(\mathrm{t})\right\}$ has stationally increments. To prove self-similarity we first note the following facts,

$$
S_{c t}=c S_{\mathrm{t}} \quad \text { and } \quad \mu_{\beta}\left(c S_{\mathrm{t}}\right)=c^{\beta} \mu_{\beta}\left(S_{\mathrm{t}}\right) \quad \text { for any } c>0 .
$$

Let us calculate the $n$-dimensional characteristic function of the process $\left\{X_{\alpha, \beta}(\mathrm{t})\right\}$;

$$
\begin{aligned}
E\left[\exp \left(i \sum_{j=1}^{n} z_{j} X_{\alpha, \beta}\left(c \mathrm{t}_{j}\right)\right)\right. & =E\left[i \sum z_{j} Y_{\alpha}\left(S_{c t_{j}}\right)\right] \\
& =\prod_{A \subset\{1, \cdots, n\}} \exp \left(-\mu_{\beta}\left(S_{c, A}\right)\left|\sum_{j \in A} z_{j}\right|^{\alpha}\right)
\end{aligned}
$$

(here $S_{c, A}$ denotes the set $\left.\left(\bigcap_{j \in A} S_{c t_{j}}\right) \cup\left(\cap_{k \oplus A}{ }^{c} S_{c t_{k}}\right)\right)$

$$
\begin{aligned}
& =\prod_{A} \exp \left(-\left.\left.\mu_{\beta}\left(S_{1, A}\right)\left|\sum_{j}\right| c\right|^{\beta / \alpha} z_{j}\right|^{\alpha}\right) \\
& =\mathbf{E}\left[\exp \left(i \sum z_{j}|c|^{\beta / \alpha} X_{\alpha, \beta}\left(t_{j}\right)\right)\right] .
\end{aligned}
$$

This implies that $\left\{X_{\alpha, \beta}(\mathbb{t})\right\}$ is an $(\alpha, \beta / \alpha)$-process.

q.e.d.

N.B. Our process $\left\{X_{\alpha, \beta}(\mathrm{t})\right\}$ satisfies the following condition stronger than ii);

ii') $\left\{X_{\alpha, \beta}(t)\right\}$ has stationary increments under the action of Euclidean solid motion. That is, $\left\{X_{\alpha, \beta}^{g}(\mathfrak{t}) \equiv X(g \mathfrak{t})-X(g \mathbb{D}) ; \mathfrak{t} \in \mathbf{R}^{d}\right\}$ has the same finite dimensional distribution as $\left\{X_{\alpha, \beta}(\mathfrak{t})\right\}$. This fact is proved as in the same manner used in the proof of ii). 


\section{§4. Comparison with fractional stable process}

As we mentioned in $\S 1$, there exists another example of $(\alpha, H)$-process $\left\{\Delta_{H}(\mathbb{t}) ; \mathfrak{t} \in \mathbf{R}^{d}\right\}$ called fractional stable process for $0<H<1$. Let us recall the definition:

$$
\Delta_{H}(\mathfrak{t} ; \omega) \equiv ? \int_{\mathbf{R}^{d}} F(\mathfrak{t}, \mathrm{x}) d Z_{\alpha}(\mathbf{x} ; \omega)
$$

where

$$
F(\mathfrak{t}, \mathrm{x}) \equiv|\mathbf{x}|^{H-(d / \alpha)}-|\mathrm{x}-\mathfrak{t}|^{H-(d / \alpha)}, \quad \mathfrak{t}, \mathrm{x} \in \mathbf{R}^{d} .
$$

Hence, we have two examples of $(\alpha, H)$-processes for a pair of parameter $(\alpha, H)$ in the range $0<H<\min (1,1 / \alpha), \alpha<2$.

A natural question pops up.

\section{"Are these processes equivalent to each other?"}

If $\alpha=2$, the answer is Yes. But the Gaussian case is exceptional. If $\alpha \neq 2$, the answer is "No". In fact these processes have different 2dimensional joint distributions. To see this, we present an argument due to Y. Sato (see [11] for the details).

Let us take two points $\mathbf{s}$ and $\mathbf{t}$ in $\mathbf{R}^{d}$ and let us calculate the 2dimensional joint characteristic functions. For our process $\left\{X_{\alpha, \beta}\right\}$ the characteristic function is

$$
\begin{aligned}
& E\left[\exp \left\{i\left\{z_{1} X_{\alpha, \beta}(\mathrm{s})+z_{2} X_{\alpha, \beta}(\mathfrak{t})\right)\right\}\right] \\
& \quad=\exp -\left\{\mu_{\beta}\left(S_{\mathrm{s}} \cap{ }^{c} S_{\mathfrak{t}}\right)\left|z_{1}\right|^{\alpha}+\mu_{\beta}\left({ }^{c} S_{\mathrm{s}} \cap S_{\mathfrak{t}}\right)\left|z_{2}\right|^{\alpha}+\mu_{\beta}\left(S_{\mathrm{s}} \cap S_{\mathfrak{t}}\right)\left|z_{1}+z_{2}\right|^{\alpha}\right\} .
\end{aligned}
$$

And for the fractional stable process $\left\{\Delta_{H}(\mathfrak{t}) ; \mathfrak{t} \in \mathbf{R}^{d}\right\}$ is

$$
E\left[\exp \left\{i\left(z_{1} \Delta_{H}(\mathrm{~s})+z_{2} \Delta_{H}(\mathrm{t})\right)\right\}\right]=\exp -\int_{\mathbf{R}^{d}}\left|F(\mathrm{~s}, \mathrm{x}) z_{1}+F(\mathrm{t}, \mathrm{x}) z_{2}\right|^{\alpha} d \mathrm{x} .
$$

To clarify the difference between (14) and (15), let us calculate the corresponding spherical components of the 2-dimensional Lévy measures.

In general, the characteristic function of 2-dimensional symmetric $\alpha$-stable distribution $\varphi$ has the unique canonical form

$$
\varphi\left(z_{1}, z_{2}\right)=\exp -k \int_{\left(\xi_{1}, \xi_{2}\right) \in S_{1}}\left|\xi_{1} z_{1}+\varepsilon_{2} z_{2}\right|^{\alpha} \lambda(d \xi),
$$

where $\lambda$ is a symmetric probability measure on the unit circle $S^{1}$. 
It is obvious that in case of the process $\left\{X_{\alpha, \beta}\right\}$ the measure $\lambda$ is concentrated on 6 points $( \pm 1,0),(0, \pm 1)$ and $( \pm \sqrt{2} / 2, \pm \sqrt{2} / 2)$. In case of the process $\left\{\Delta_{H}(\mathbf{t}) ; \mathbf{t} \in \mathbf{R}^{d}\right\}$

$$
\int_{\mathbf{R}^{d}}\left|F(\mathrm{~s}, \mathrm{x}) z_{1}+F(\mathrm{t}, \mathrm{x}) z_{2}\right|^{\alpha} d \mathrm{x}=\int_{\mathbf{R}^{d}}\left|\xi_{1}(\mathrm{~s}, \mathrm{t} ; \mathrm{x}) z_{1}+\xi_{2}(\mathrm{~s}, \mathrm{t} ; \mathrm{x}) z_{2}\right|^{\alpha} \Xi(\mathrm{s}, \mathrm{t} ; \mathrm{x}) d \mathrm{x}
$$

where we set

$$
\begin{aligned}
& E(\mathrm{~s}, \mathrm{t} ; \mathrm{x})=\left|F^{2}(\mathrm{~s}, \mathrm{x})+F^{2}(\mathrm{t}, \mathrm{x})\right|^{\alpha / 2}, \\
& \xi_{1}(\mathrm{~s}, \mathrm{t} ; \mathrm{x})=F(\mathrm{~s}, \mathrm{x}) /\left(F^{2}(\mathrm{~s}, \mathrm{x})+F^{2}(\mathrm{t}, \mathrm{x})\right)^{1 / 2} \text { and } \\
& \xi_{2}(\mathrm{~s}, \mathrm{t} ; \mathrm{x})=F(\mathrm{t}, \mathrm{x}) /\left(F^{2}(\mathrm{~s}, \mathrm{x})+F^{2}(\mathrm{t}, \mathrm{x})\right)^{1 / 2} .
\end{aligned}
$$

Recall the fact that $F(\mathrm{t}, \mathrm{x})$ is continuous in $\mathrm{t}$ and $\mathrm{x}$ and $\Xi(\mathrm{s}, \mathrm{t} ; \mathrm{x})=0$ only a set of Lebesgue measure 0 if $\mathrm{s} \neq \mathrm{t}$. The measure $\lambda$ on $S^{1}$ is the image of the measure $\Xi(\mathrm{s}, \mathrm{t} ; \mathrm{x}) d \mathbf{x}$ on $\mathbf{R}^{d}$ by the map $\left(\xi_{1}, \xi_{2}\right)$. It is easy to see that the measure $\lambda$ has absolute continuous part. Therefore, we have

TheOREM 5 ([11]). The two $(\alpha, H)$-processes $\left\{X_{\alpha, \alpha H}(\mathfrak{t}) ; \mathfrak{t} \in \mathbf{R}^{d}\right\}$ and $\left\{\Delta_{H}(\mathfrak{t}) ; \mathfrak{t} \in \mathbf{R}^{d}\right\}$ for $H<\min (1,1 / \alpha)$ and $0<\alpha<2$, are not equivalent to each other.

\section{§ 5. Generalized Chentsov type representation and conjugate Gaussian process}

As we saw in $\S 3$ and $\S 4$, the processes which are constructed by integral-geometric method make a special class of stochastic processes. In this section we investigate some properties of this class. At first let us clarify the object in which we are concerned:

Definition. A random field $\left\{X(\mathfrak{t}) ; \mathfrak{t} \in \mathbf{R}^{a}\right\}$ is called a process of generalized Chentsov type if there exist a measure space $(E, \mathscr{B}, \mu)$ and a map

$$
S: M \longrightarrow \mathscr{B}
$$

such that the process $\left\{X(t) ; t \in \mathbf{R}^{d}\right\}$ is written as

$$
X(\mathrm{t})=Y\left(S_{\mathrm{t}}\right),
$$

where $\mathscr{Y}=\{Y(B) ; B \in \mathscr{B}, \mu(B)<\infty\}$ is a random stable measure corresponding to $(E, \mathscr{B}, \mu)$.

It is obvious that our $(\alpha, H)$-processes are examples of processes of 
generalized Chentsov type.

5-1. Let us fix an integer $n>d$. Let $\left\{X_{\alpha, \beta}^{n}(\tilde{\mathbb{t}}) ; \tilde{\mathfrak{t}} \in \mathbf{R}^{n}\right\}$ be the $n$ parameter $(\alpha, H)$-process that we constructed in $\S 3$. Let us restrict the parameter $\tilde{\mathfrak{t}}$ to a linear subspace $\mathbf{R}^{d} \subset \mathbf{R}^{n}$, and consider the $d$-parameter process; $\left\{\left.X^{d}(\mathbb{t}) \equiv X^{n}\right|_{\mathbf{R}^{d}}(\mathfrak{t}), \mathbb{t} \in \mathbf{R}^{d}\right\}$.

This process $\left\{X^{d}\right\}$ seems a new $d$-parameter $(\alpha, H)$-process of generalized Chentsov type. Let us check that this process is really new or not.

To simplify calculation let us take $n=d+1$ and assume $\mathbf{R}^{d+1}=$ $\mathbf{R}^{d} \times \mathbf{R}^{1}$. The set $S_{\mathfrak{t}}^{n}, \mathfrak{t} \in \mathbf{R}^{d}$, is

$$
\begin{gathered}
\left\{\left(\mathrm{x}, x_{n}, x_{0}\right) ;\|\mathrm{x}\|^{2} \leq x_{0}^{2}-x_{n}^{2}, \mathrm{x} \in \mathbf{R}^{d}\right\} \triangle\left\{\left(\mathrm{x}, x_{n}, x_{0}\right) ;\|\mathrm{x}-\mathrm{t}\|^{2} \leq x_{0}^{2}-x_{n}^{2}\right\} \\
=\left\{\left(\mathrm{x}, x_{n}, x_{0}\right) ;\|\mathrm{x}\| \leq \eta_{0}\right\} \triangle\left\{\left(\mathbf{x}, x_{n}, x_{0}\right) ;\|\mathrm{x}-\mathbf{t}\| \leq \eta_{0}\right\} \\
\text { where } \eta_{0}=\left(x_{0}^{2}-x_{n}^{2}\right)^{1 / 2} .
\end{gathered}
$$

Set $\sin \theta=\frac{x_{n}}{x_{0}}$ and $\eta_{0}=x_{0} \cos \theta$, then the set above is rewritten as

$$
\left\{\left(\mathbf{x}, \eta_{0}, \theta\right) ;\|\mathbf{x}\| \leq \eta_{0},|\theta| \leq \frac{\pi}{2}\right\} \triangle\left\{\left(\mathbf{x}, \eta_{0}, \theta\right) ;\|\mathbf{x}-\mathbf{t}\| \leq \eta_{0}\right\}
$$

That is, $S_{\mathrm{t}}^{n}$ is considered as $S_{\mathrm{t}}^{d} \times[-\pi / 2, \pi / 2]$ by the parameter $\left(\eta_{0}, \theta\right)$. This Jacobian $\partial\left(x_{n}, x_{0}\right) / \partial\left(\eta_{0}, \theta\right)$ is equal to $x_{0}$. Then the quantity $\mu_{\beta}^{n}\left(S_{\mathfrak{t}}^{n}\right)$ is evaluated as follows:

$$
\begin{aligned}
\mu_{\beta}^{n}\left(S_{\mathrm{t}}^{n}\right) & =\int_{\mathbf{R}^{n} \times \mathbf{R}_{+}} \chi_{S_{\mathbf{t}}^{n}}\left(\mathbf{x}, x_{n}, x_{0}\right) x_{0}^{\beta-1-n} d x_{0} d \mathbf{x} d x_{n} \\
& =\int_{\pi / 2}^{\pi / 2} \int_{\mathbf{R}^{d} \times \mathbf{R}_{+}} \chi_{S_{\mathfrak{t}}^{d}}\left(\mathbf{x}, \eta_{0}\right) \eta_{0}^{\beta-1-d} d \eta_{0} d \mathbf{x}^{\cos ^{d+1-\beta}} \theta d \theta \\
& =k \mu_{\beta}^{d}\left(S_{\mathrm{t}}^{d}\right), \quad \text { where } k=\int_{\pi / 2}^{\pi / 2} \cos ^{d+1-\beta} \theta d \theta
\end{aligned}
$$

The same calculation holds for any set $S_{1, A}$ which characterizes $n$-dimensional distribution of the process (see the proof of Theorem 4). Thus we have

Proposition 6. For any integers $n$ and $d, n \geq d$, there exist a constant $k$ such that $\left\{X_{\alpha, \beta}^{n}{\mid \mathbb{R}^{d}}_{(t)} ; \mathfrak{t} \in \mathbf{T}\right\}$ and $\left\{k \cdot X_{\alpha, \beta}^{d}(\mathfrak{t}) ; \mathfrak{t} \in \mathbf{T}\right\}$ have the same finite dimensional distributions, that is, the two processes are essentially the same.

\section{5-2. Conjugate class and Conjugate Gaussian process}

Let us proceed to consider a general classes of stochastic processes 
of generalized Chentsov type with parameter in $\mathbf{T}$. The parameter space $\mathbb{T}$ is not necessarily the Euclidean space and the measure space $\mathbb{E}$ is not assumed to be the set of spheres.

Fix a measure $\mu$ on $\mathbb{E}$ and a family $\left\{S_{\mathfrak{t}} ; \mathfrak{t} \in \mathbf{T}\right\}$. Specifying the random measure $\mathscr{Y}_{\alpha}=\left\{Y_{\alpha}(\cdot)\right\}$ to an $\alpha$-stable random measure corresponding to $(\mathbb{E}, \mu)$, we have a process $\left\{X_{\alpha}(\mathfrak{t}) ; \mathfrak{t} \in \mathbf{T}\right\}$ by

$$
X_{\alpha}(\mathrm{t}) \equiv Y_{\alpha}\left(S_{\mathrm{t}}\right) \text {. }
$$

Definition. The set of the processes $\left\{\left\{X_{\alpha}(\mathfrak{t}) ; \mathfrak{t} \in \mathbf{T}\right\} ; 0<\alpha \leq 2\right\}$ is called a conjugate class and the Gaussian element $\left\{X_{2}(t)\right\}$ is called the conjugate Gaussian process to any other element $\left\{X_{\alpha}(\mathbb{t})\right\} 0<\alpha<2$.

Proposition 7. A quasi-metric $d$ on $\mathbf{T}$ is induced from the Hausdorff metric on the subsets of $E$ with respect to the measure $\mu$;

$$
d(\mathbb{t}, \mathrm{s}) \equiv \mu\left(S_{\mathrm{t}} \triangle S_{\mathrm{s}}\right) .
$$

That is, the difference $X_{2}(\mathrm{t})-X_{2}(\mathrm{~s})$ of the conjugate Gaussian process is subject to the Gaussian law of mean zero and variance $d(\mathfrak{t}, \mathrm{s})$. Such a process is called a Lévy's Brownian motion with parameter in the metric space $(\mathbf{T}, d)$ (see [7]).

Using the above proposition we have

Theorem 8 (cf. [12] of the Gaussian case). There is no symmetric $\alpha$ stable $H$-self-similar process with stationary increments of generalized Chentsov type for $H>1 / \alpha$.

Proof. Let $\left\{X_{\alpha}(\mathbb{t})\right\}$ be an symmetric $\alpha$-stable process with stationary increments of generalized Chentsov type. Then the conjugate Gaussian process $\left\{X_{2}(\mathfrak{t})\right\}$ is a $\alpha H / 2$-self-similar Gaussian process with stationary increments. The corresponding metric in $R^{d}$ is $|\mathfrak{t}-\mathbf{s}|^{\alpha H}$. For $H>1 / \alpha$ this is not a metric function on $\mathbf{R}^{d}$.

q.e.d.

Fix a conjugate class $\left\{\left\{X_{\alpha}(\mathfrak{t}) ; \mathfrak{t} \in \mathbf{T}\right\} ; 0<\alpha \leq 2\right\}$ and consider the 2dimensional characteristic functions

$$
\begin{aligned}
& \mathbf{E}\left[\exp i\left\{X_{\alpha}(\mathrm{s}) z_{1}+X_{\alpha}(\mathbf{t}) z_{2}\right\}\right] \\
& \quad=\exp -\left\{\mu\left(S_{\mathrm{s}} \cap{ }^{c} S_{\mathrm{t}}\right)\left|z_{1}\right|^{\alpha}+\mu\left({ }^{c} S_{\mathrm{s}} \cap S_{\mathrm{s}}\right)\left|z_{2}\right|^{\alpha}+\mu\left(S_{\mathrm{s}} \cap S_{\mathrm{t}}\right)\left|z_{1}+z_{2}\right|^{\alpha}\right\} .
\end{aligned}
$$

Hence, the characteristic function of $\left\{X_{\alpha}\right\}$ is determined by the scalars $\mu\left(S_{s} \cap{ }^{C} S_{t}\right), \mu\left({ }^{C} S_{s} \cap S_{t}\right)$ and $\mu\left(S_{s} \cap S_{t}\right)$. 
Let us consider the conjugate Gaussian process

$$
\mathbf{E}\left[\exp i\left\{X_{2}(\mathrm{~s}) z_{1}+X_{2}(\mathrm{t}) z_{2}\right\}\right]=\exp -\left\{\frac{1}{2} \sigma_{\mathrm{s}} z_{1}^{2}+\sigma_{\mathrm{st}} z_{1} z_{2}+\frac{1}{2} \sigma_{\mathrm{t}} z_{2}^{2}\right\},
$$

where $\sigma_{\mathrm{s}}, \sigma_{\mathrm{t}}$ and $\sigma_{\mathrm{st}}$ are the variances and the covariance of the process $\left\{X_{2}\right\}$. On the other hand, the same characteristic function has the following form,

$$
\exp -\left\{\mu\left(S_{\mathrm{s}} \cap{ }^{c} S_{\mathrm{t}}\right)\left|z_{1}\right|^{2}+\mu\left({ }^{c} S_{\mathrm{s}} \cap S_{\mathrm{t}}\right)\left|z_{2}\right|^{2}+\mu\left(S_{\mathrm{s}} \cap S_{\mathrm{t}}\right)\left|z_{1}+z_{2}\right|^{2}\right\} .
$$

The quantities $\mu\left(S_{s} \cap{ }^{c} S_{\mathrm{t}}\right)$ etc. are uniquely determined by $\sigma_{\mathrm{s}}, \sigma_{\mathrm{t}}$ and $\sigma_{\mathrm{st}}$ as

$$
\begin{gathered}
\mu\left(S_{\mathrm{s}} \cap{ }^{c} S_{\mathrm{t}}\right)=\frac{1}{2}\left(\sigma_{\mathrm{s}}-\sigma_{\mathrm{st}}\right), \quad \mu\left({ }^{c} S_{\mathrm{s}} \cap S_{\mathrm{t}}\right)=\frac{1}{2}\left(\sigma_{\mathrm{s}}-\sigma_{\mathrm{st}}\right) \\
\text { and } \mu\left(S_{\mathrm{s}} \cap S_{\mathrm{t}}\right)=\frac{1}{2} \sigma_{\mathrm{st}} .
\end{gathered}
$$

Thus, we have the following determinism of the symmetric $\alpha$-stable processes of generalized Chentsov type.

Theorem 9. Let $\left\{\left\{X_{\alpha}(\mathrm{t}) ; \mathrm{t} \in \mathbf{T}\right\}, 0<\alpha \leq 2\right\}$ and $\left\{\left\{X_{\alpha}^{\prime}(\mathrm{t}) ; \mathrm{t} \in \mathbf{T}\right\}\right\}$ be two conjugate classes. If the Gaussian elements $\left\{X_{2}(\mathrm{t})\right\}$ and $\left\{X_{2}^{\prime}(\mathrm{t})\right\}$ are subject to the same law, then, for any $0<\alpha<2$, the processes $\left\{X_{\alpha}(\mathrm{t})\right\}$ and $\left\{X_{\alpha}^{\prime}(\mathrm{t})\right\}$ have common 2-dimensional distributions. Especially any $(\alpha, H)$-processes of generalized Chentsov type for fixed $\alpha$ and $H$ have the same 2-dimensional distributions up to the normalizing factors.

\section{REFERENCES}

[1] Bochner, S., Harmonic Analysis and the Theory of Probability, 1955, Univ. of California Press.

[2] Cambanis, S. and Maejima, M., Two classes of self-similar stable processes with stationary increment, Stoch. Proc. Appl., 32 (1989), 305-329.

[ 3 ] Chentsov, N. N., Lévy's Brownian motion of several parameters and generalized white noise, Theory Probab. Appl., 2 (1957), 265-266.

[ 4 ] Kasahara, Y. and Maejima, M., Weighted sums of i.i.d. random variables attracted to integrals of stable processes, Probab. Th. Rel. Fields, 78 (1988), 75-96.

[ 5 ] Kasahara, Y., Maejima, M. and Veraat, W., Log-fractional stable processes, Stochastic Process. Appl., 30 (1988), 329-339.

[ 6 ] Kojo, K., and Takenaka, S., On canonical representations of stable $M(t)$-processes, preprint (1990).

[ 7 ] Lévy, P., Processus stochastiques et mouvement brownien, Gauthier-Villers, 1965.

[ 8 ] Maejima, M., On a class of self-similar processes, Z. Wahrsch. Verw. Gebiete, 62 (1983), 53-72.

[9] —-, A remark on self-similar processes with stationary increments, Canad. J. Statist., 14 (1986), 81-82. 
[10] Mandelbrot, B. B. and van Ness, J. W., Fractional Brownian motions, fractional noises and applications, SIAM Rev., 10 (1968), 422-437.

[11] Sato, Y., Joint distributions of some self-similar stable processes, preprint (1989).

[12] Takenaka, S., Representation of Euclidean random fields, Nagoya Math. J., 105 (1987), 19-31.

[13] Taqqu, M. S. and Wolpert, R., Infinite variance self-similar processes subordinated to a Poisson measure, Z. Warsch. verw. Gebiete, 62 (1983), 53-72.

[14] Vervaat, W., Sample path properties of self-similar processes with stationary increments, Ann. Probab., 13 (1985), 1-27.

Department of Mathematics

Hiroshima University

Kagamiyama 1

Higashi-Hiroshima 742

Japan 